\title{
Power line communication network trial and management in Korea
}

\author{
Jae-Jo Lee ${ }^{1}$, Choong Seon Hong ${ }^{2}$, Joon-Myung Kang ${ }^{*,+3}$ and James Won-Ki Hong ${ }^{3}$ \\ ${ }^{1}$ Electro-Fusion Research Division, Korea Electrotechnology Research Institute (KERI), Uiwang, Korea \\ ${ }^{2}$ Department of Computer Engineering, Kyung Hee University, Seoul, Korea \\ ${ }^{3}$ Department of Computer Science and Engineering, POSTECH, Pohang, Korea
}

\begin{abstract}
SUMMARY
Power line communication (PLC) has become an important network access technology to conveniently and effectively provide various useful services such as remote meter reading, broadband Internet access, and home networking services. As the deployment and use of PLC networks increase, it is essential that we need to be able to configure, monitor, and control the PLC network resources. In this paper, we present an overview of PLC network technology and describe a trial PLC network and its deployment in Korea. We then present the design and implementation of a network management system for PLC networks. The experience on the deployment and management of the trial PLC network is also presented. Copyright (c) 2006 John Wiley \& Sons, Ltd.
\end{abstract}

\section{INTRODUCTION}

Power line communication (PLC) has received tremendous attention in recent years as an alternative and cost-effective last-mile-access technology [1,2]. It is easy to install PLC networks cost-effectively because the power lines already exist everywhere. Thus, power lines are frequently regarded as the preferred medium for providing a broadband connection to rural or remote areas where telephone and cable connections do not exist. PLC can provide data services, such as broadband Internet service, VoIP service, and a variety of value-added services, such as remote metering, street light control, home security, home appliance control, and many more. Efforts are also underway to provide the monitoring and control capabilities of their own power line equipment and facilities. A number of PLC systems have been installed worldwide for various kinds of field trials to demonstrate its feasibility and effectiveness [3,4].

Standardization effort on PLC networks has been ongoing by many national and international standards organizations and industrial forums. The majority of the work has been focusing on the physical and MAC layer aspects. IEEE P1675 [5] primarily deals with the hardware associated with the distribution lines that the PLC signal is being injected into. IEEE P1901 [6] deals with the standard for high-speed (i.e., $>100 \mathrm{Mb} / \mathrm{s}$ at the physical layer) communication devices via alternating current electric power lines. IEEE P1775 [7] deals with PLC equipment, electromagnetic compatibility requirements, and testing and measurement methods. OPERA (Open PLC European Research Alliance) [8] is a European, multiorganization R\&D project which aims to standardize PLC systems and to develop business plans and procedures for network maintenance as well as service provisioning, along with market research to understand the requirements of end users. The Universal Powerline Association (UPA) [9] has attracted membership from PLC companies, utilities, electronic equipment manufacturers, and chipset providers to catalyze the growth of PLC technology by delivering UPA certified products that comply with agreed specifications.

*Correspondence to: Joon-Myung Kang, Department of Computer Science and Engineering, POSTECH, San 31 Hyoja, Namgu, Pohang, Korea.

${ }^{\dagger}$ E-mail: eliot@postech.ac.kr 
As the standardization effort has been achieved, vendors such as Xeline [10], DS2 [11], Intellon [12], and others have been developing PLC chips and devices to enable the deployment of PLC networks and thus to provide various services based on PLC networks. Recently, Xeline and DS2 were successful in developing PLC chips that can support a transmission speed of $200 \mathrm{Mb} / \mathrm{s}$, which can be used to support a variety of multimedia applications, such as voice, audio, video, and TV.

Like any other types of network, PLC networks need to be monitored and controlled in order to provide secure, efficient and reliable services. Although the physical characteristics differ much from existing access networks, such as xDSL and cable modem, PLC is not an entirely different type of network when it comes to network management. Thus, we can utilize much of the already existing network management techniques, tools and protocols for managing PLC networks.

Recently, a pilot PLC network using medium- and low-voltage power lines has been deployed by the Korea Electrotechnology Research Institute (KERI) over a distance of approximately $5 \mathrm{~km}$ in the Chunggae area, which is in a suburb of Euiwang city near Seoul, Korea. Some useful services, such as broadband Internet service, automatic metering service and VoIP, have been used by 20 household subscribers. A PLC network management system has also been developed to manage this pilot PLC network. This paper presents the deployment of a trial PLC network, the network management system we have developed for it, and the experience on the trial.

The remainder of this paper is organized as follows. Section 2 presents a brief overview of PLC networking technology. Section 3 describes the deployed PLC trial network in Korea. Section 4 presents the design of a PLC NMS (network management system). Section 5 presents the implementation details of PLC NMS. Section 6 presents the field test result of PLC NMS applied to the pilot network in Korea. Finally, conclusions are drawn and future work is discussed in Section 7.

\section{OVERVIEW OF PLC NETWORK TECHNOLOGY}

PLC technology enables utility companies to deploy a communications network over existing power line infrastructures $[3,4]$ by transmitting data signals through the same power cables that transmit electricity. This technology, however, uses a different frequency from the power cable. PLC can be used for various network services such as broadband Internet access, telephony, remote metering, and home networking services. There are largely two types of PLC networks depending on the area they cover: access PLC and in-home PLC. Access PLC is also called broadband over power line (or BPL) [10].

The in-home PLC network uses the existing electrical wiring and outlets in a house or an office to connect PCs, broadband modems, set-top boxes, gaming consoles, audio/video players, security cameras, and electronic home appliances. As a result, every electrical outlet becomes an Ethernet jack or a network connection point-without adding any new wires. Consumers can instantly install their home networks just by plugging the PLC equipment into their power plugs. Since it is so easy to set up and use, the inhome PLC network is generally self-installed by consumers. It may also be set up by service providers, such as cable, DSL, and satellite companies.

The BPL or access PLC network uses the electric utility's outside power lines to deliver broadband Internet service to homes and small businesses. Accordingly, BPL competes with other broadband Internet access services, such as cable modem and xDSL. Once the Internet service reaches the home over the outside power lines, it can be distributed throughout the house over existing electrical wiring just like the in-home PLC network. The access PLC network is generally set up and managed by electric utility companies. There are two types of access PLC technologies, which are low-voltage (LV) PLC and medium-voltage (MV) PLC. LV PLC is the communication technology that uses low-voltage power lines and MV PLC is the communication technology that uses medium-voltage power lines. For the backbone network, cable network or fiber optic communication is used since a cost-effective PLC technology for supporting long-haul communication has not yet been developed.

Figure 1 illustrates a typical PLC network, which is composed of the following components: 


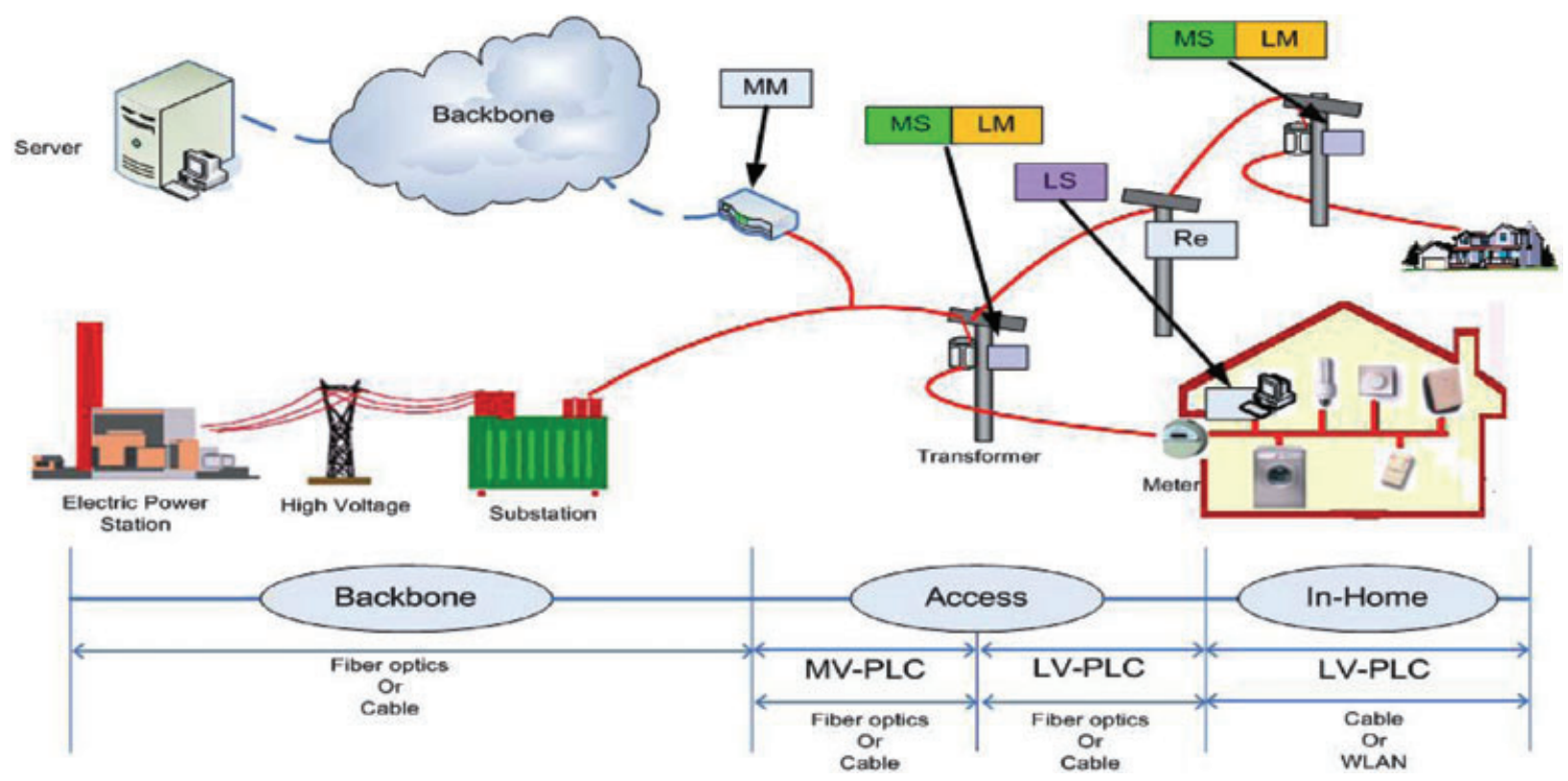

Figure 1. Overview of PLC network

- Medium-voltage master (MM): the device to connect the backbone network and MV-PLC.

- Medium-voltage slave (MS) and low-voltage master (LM): the device to transfer the signal between LVPLC and MV-PLC.

- Low-voltage slave (LS): the device to transfer the signal between LM and home devices.

- Repeater (Re): the device to amplify the signal between MV-PLC modems.

Several MSs are typically connected to an MM, which coordinates and relays communication between MSs and the backbone network. An MS is usually housed in the same physical device as LM, which coordinates and relays communication between LSs and the MV-PLC network. The repeaters are used to amplify the signal between master and slave modems.

\section{DEPLOYED PLC NETWORK IN KOREA}

In this section, we describe the deployed PLC trial network in Korea. The MV power distribution line of Korea is composed of $22.9 \mathrm{kV}$, 3-phase 4-wire, tree type, and overhead wire. Each subscriber is provided with $220 \mathrm{~V}$ electric power through the pole transformer. About 30 households are connected to a pole transformer.

Currently, the trial PLC network using the MV power distribution line has been constructed over a distance of about $5 \mathrm{~km}$ in the Chunggae area located in a suburb of Euiwang city near Seoul, Korea, as shown in Figure 2 [13]. Figure 2 presents the structure of the medium-voltage power distribution network for the construction of the MV PLC access network. In Figure 2, locations A-G are where broadband signal couplers for MV distribution lines are installed, and the distances between the locations are also marked. Especially, the section between B and C consists mostly of underground cable, while the others are overhead wire sections. To perform various experiments for high/low-voltage PLC, the PLC access network is constructed over the area where $5 \mathrm{~km}$ of MV power distribution line has been built, and the LV PLC network service for 50 households is possible. This is the first case to construct a PLC subscriber network using the MV and LV power lines in Korea. Twenty household subscribers are currently 


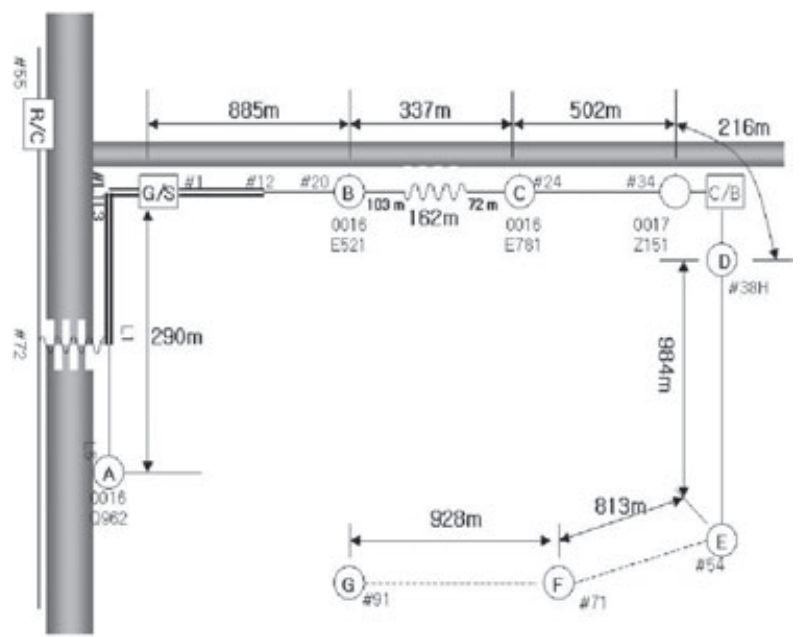

Figure 2. Configuration of medium-voltage trial PLC network

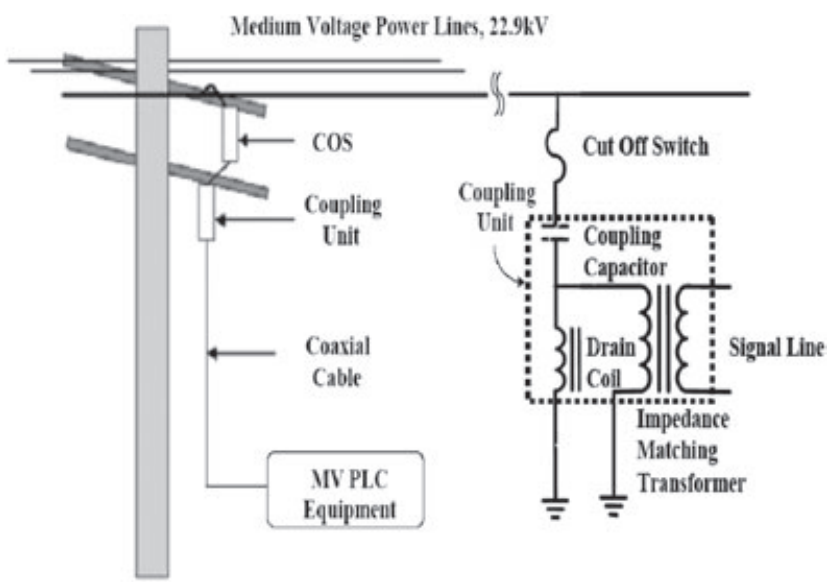

Figure 3. Signal coupling with MV coupling unit

participating in the trial through the access PLC network, which has been used efficiently for the measurement of electromagnetic interference (EMI), Internet access, and VoIP service.

For an overhead MV power line cable in the test field of PLC, an aluminum conductor steel-reinforced outdoor cross-linked polyethylene insulated wires (ACSR-OC) cable is used. The cable consists of mainly three concentric layers of six aluminum cores, a steel core of $3.5 \mathrm{~mm}$ diameter in the middle of the six aluminum cores to reinforce the aluminum cores, and a dielectric insulator of cross-linked (XL) polyethylene. Also, a wire-to-ground (WTG) arrangement is applied, as illustrated in Figure 3.

Figure 3 shows the means of coupling signals with $22.9 \mathrm{kV}$ MV power lines by using a coupling unit. The coupling unit is composed of a capacitor of $2 \mathrm{nF}$ for communication signals in the frequency band between $1 \mathrm{MHz}$ and $30 \mathrm{MHz}(<1 \mathrm{~dB}$ attenuation) and an inductor of $1 \mathrm{mH}$ in order to prevent high-voltage power of $60 \mathrm{~Hz}$ on MV power lines. Owing to harsh communication conditions of MV power lines, a cutoff-switch (COS) is used to protect the coupling unit. A coupling unit is connected to a MV-PLC modem through a coaxial cable of $75 \Omega$ characteristics impedance, which is about $13 \mathrm{~m}$ long. Figure 4 shows a photograph of overhead MV power lines and a coupling unit.

Figure 5 shows a photograph of installed MV-PLC network equipment with a surge protection circuit, MV master modem, LV master modem, EMS agent, and cable modem. The MV master modem can 


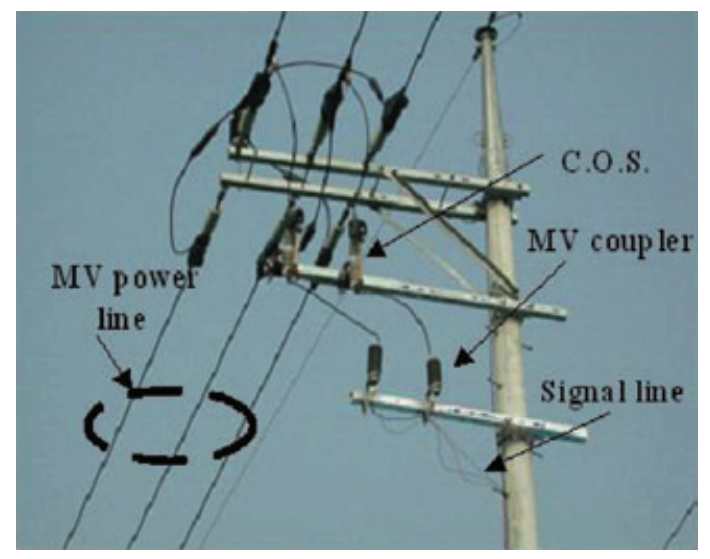

Figure 4. Installed MV coupler

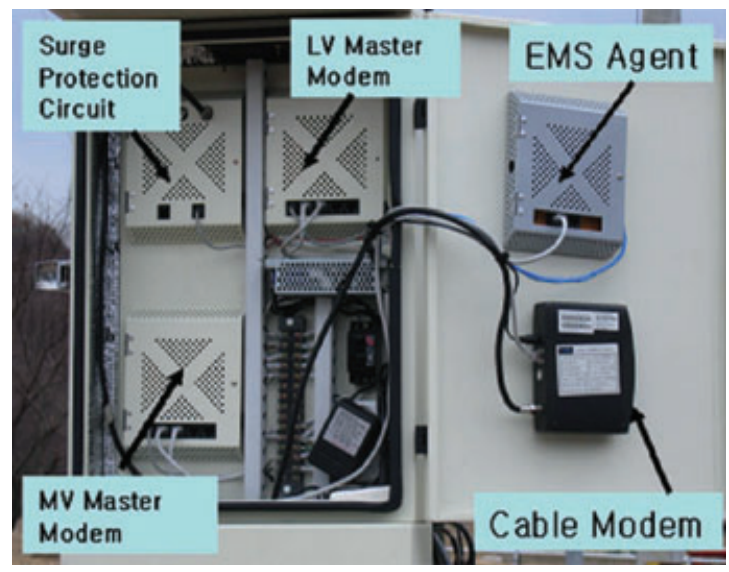

Figure 5. Configuration of MV PLC equipment

connect the PLC network to the Internet by coupling the signal from the cable modem. The PLC modems used for trial PLC network can support a data rate up to $24 \mathrm{Mb} / \mathrm{s}$ using a frequency band of $2-23 \mathrm{MHz}$. Its specifications are shown in Table 1 [10].

\section{DESIGN OF PLC NETWORK MANAGEMENT SYSTEM}

The objective for our PLC network management system (NMS) design is to create solutions for the integrated management of PLC network devices. This section presents the design of a PLC network management system, which is used to monitor and control the trial PLC network.

\subsection{Architecture of PLC NMS}

Figure 6 illustrates the architecture of the PLC network management system for managing the MV and LV PLC modems. Our PLC network management system (NMS) uses an SNMP [14] and one or more SNMP agents. Since computing resources are scarce in master and slave modems, an SNMP agent is not equipped in these devices. Instead, a separate proxy agent (i.e., EMS) has been developed to interact with the SNMP manager in the northbound and master and slave modems in the southbound for exchanging management operations and information. 


\begin{tabular}{|lll|}
\hline Item & Characteristic & Special features \\
\hline Data rate & Up to 24 Mb/s & Hierarchical MAC \\
Modulation type & DMT based on PSK & Channel scanning function \\
Number of sub-carriers & 256 & Automated routing procedure \\
Frequency band & $2-23 \mathrm{MHz}$ & Coexistence of multiple master \\
Bit loading & $0 / 1 / 2 / 3$ bits, adaptively & Programmable notch filter \\
Forward error correction & Concatenated with & Adaptive power allocation \\
codes & convolutional and RS Code & \\
Multiple access & CSMA/CA & Changeable network configuration \\
Topology & Master/slave & 56-bit DES encryption \\
CMOS Technology & $0.18 \mu \mathrm{m}$ & Half duplex by time division \\
Package & $100-$ pin TQFP package & \\
& $\left(14 \times 14 \mathrm{~mm}^{2}\right)$ & \\
\hline
\end{tabular}

Table 1. Specifications of the deployed PLC modem

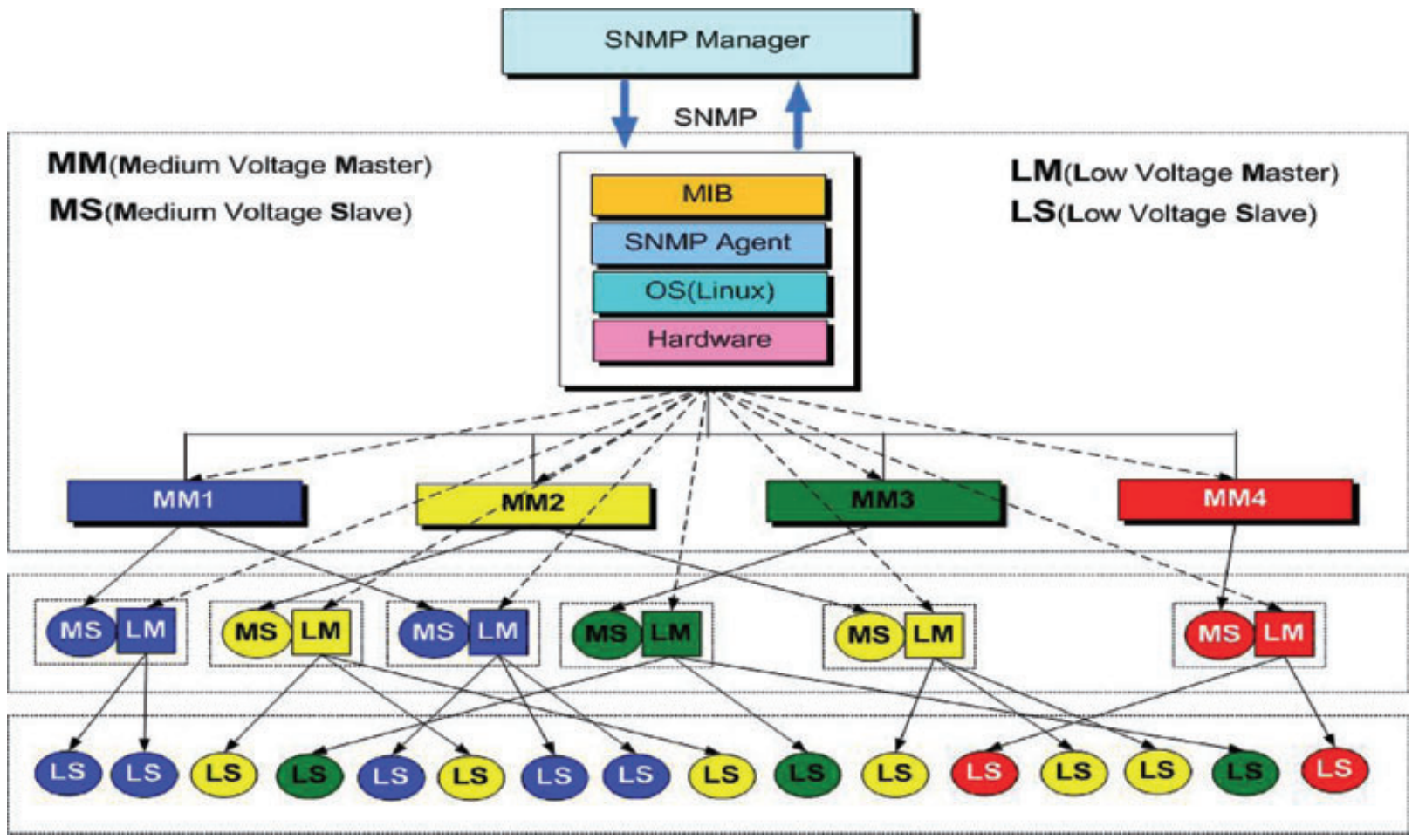

Figure 6. Architecture of PLC network management system

Note that each modem has a master/slave configuration. Hence, a master modem internally gathers management information from one or more slave modems which have the same group ID regardless of their physical locations, and the SNMP agent manages one or more master modems. The agent provides management information of network elements to the SNMP manager and uses a broadcasting message in medium access control (MAC) level to the master modems. The modems then reply with their location information to the agent. Essentially, the manager simply communicates with the agent on a periodic and on-demand basis to gather MIB information and to control the elements. The network managed by the PLC NMS could include MV modem, LV master/slave modem, and other PLC network elements, such as home gateway. In this paper, we only describe the architecture for managing PLC modems as network elements. 


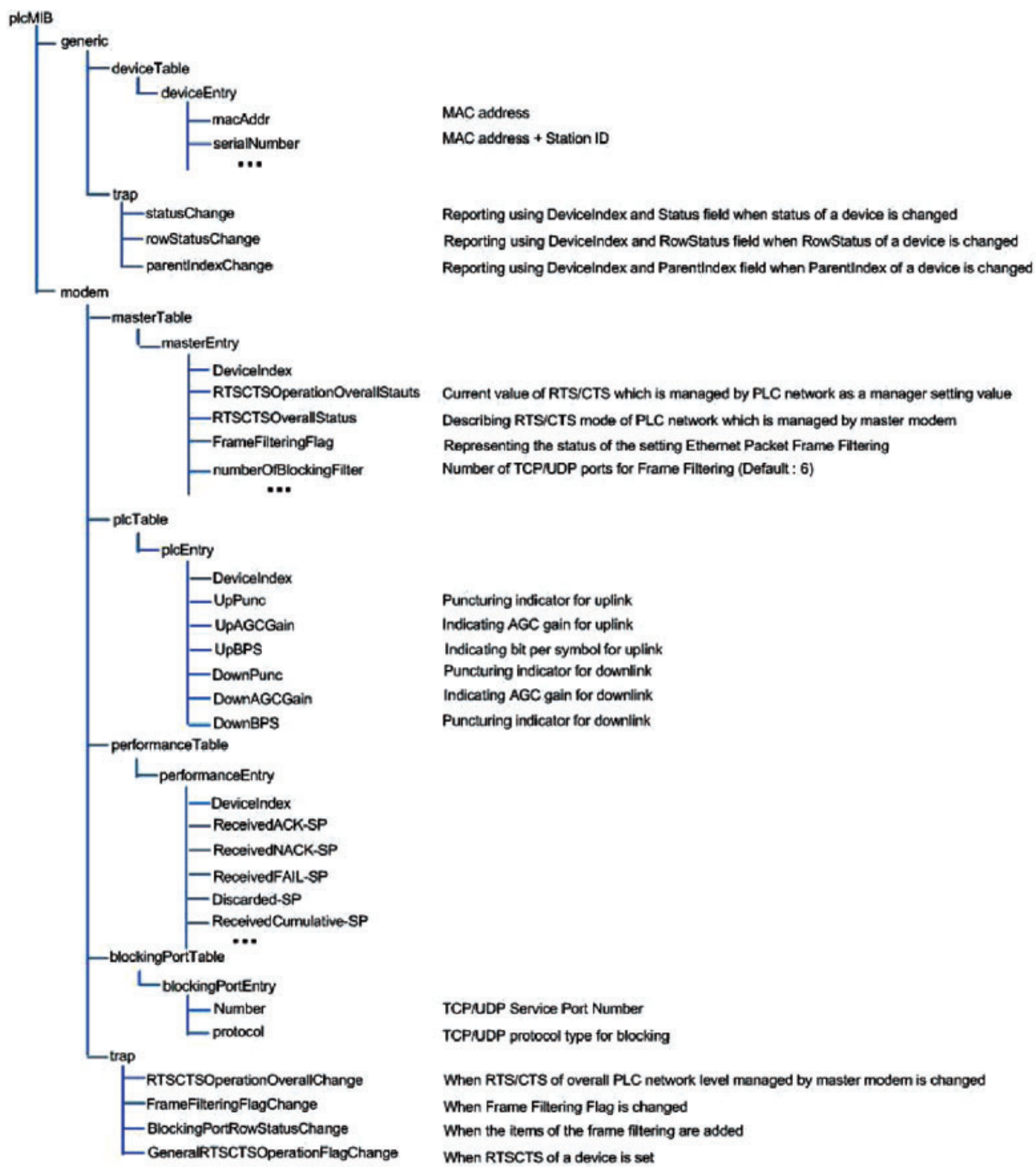

Figure 7. Definition of PLC-MIB

\subsection{PLC MIB}

The PLC MIB has been defined as an enterprise MIB consisting of generic MIB and PLC modem's MIB as illustrated in Figure 7. The generic MIB in our design consists of device and trap tables, and mainly contains the configuration information of PLC modems. The index field in an agent acts as anique 
primary key which is used to find tables for the same device. This index field is made of the rightmost three octets of the master modem's MAC address and station ID (SID). For example, if the master modem's MAC address is '00 0c $93140011^{\prime}$ and SID is 4, the index field is 335548676, which is the decimal value of $0 \times 11001404$. We assign SID 0 to the master, 1-3 to the repeaters, and 4-31 to the slaves by the configuration of the modem's DIP switch, which has 3-bit group ID and 5-bit station ID. The status field shows the current state of the modem as act (1), fault (2), blank (3), reset (4), and data error (5).

The PLC modem MIB is composed of a general table, a master table, a PLC table, a performance table and a trap table. The general table has information concerning the group of master modems. The master table has information concerning the registered master modems. Moreover, the PLC table has the fundamental management information of PLC networks. We can obtain and analyze various information on the PLC networks through this table. Finally, the performance table has information concerning the repeaters and the slave modems except for the master modems which are registered in the general table. Therefore, the performance information of the master modems can be calculated using the performance information of the slave modems, which are managed by the master modems. All values which are managed by the performance table have the Counter32 type, except DeviceIndex. For the purpose of security, we prepare the blocking port table, which has the secure port number, transport protocol to be used, and row status fields in addition to DeviceIndex. Using this table, we can check whether the customer's own devices, such as personal computers, are disclosed on the network or not. This table is set by an NMS operator.

\subsection{Architectural design of PLC NMS}

This section describes the architectural design of the PLC network management system from the viewpoint of manager and agent. The architecture of our PLC NMS is illustrated in Figure 8. The administrator can manage the PLC networks by using the NM application client or web browser by connecting to the NMS server, which is composed of a front-end manager, configuration manager, fault manager, performance manager, security manager, DB engine, and SNMP engine.

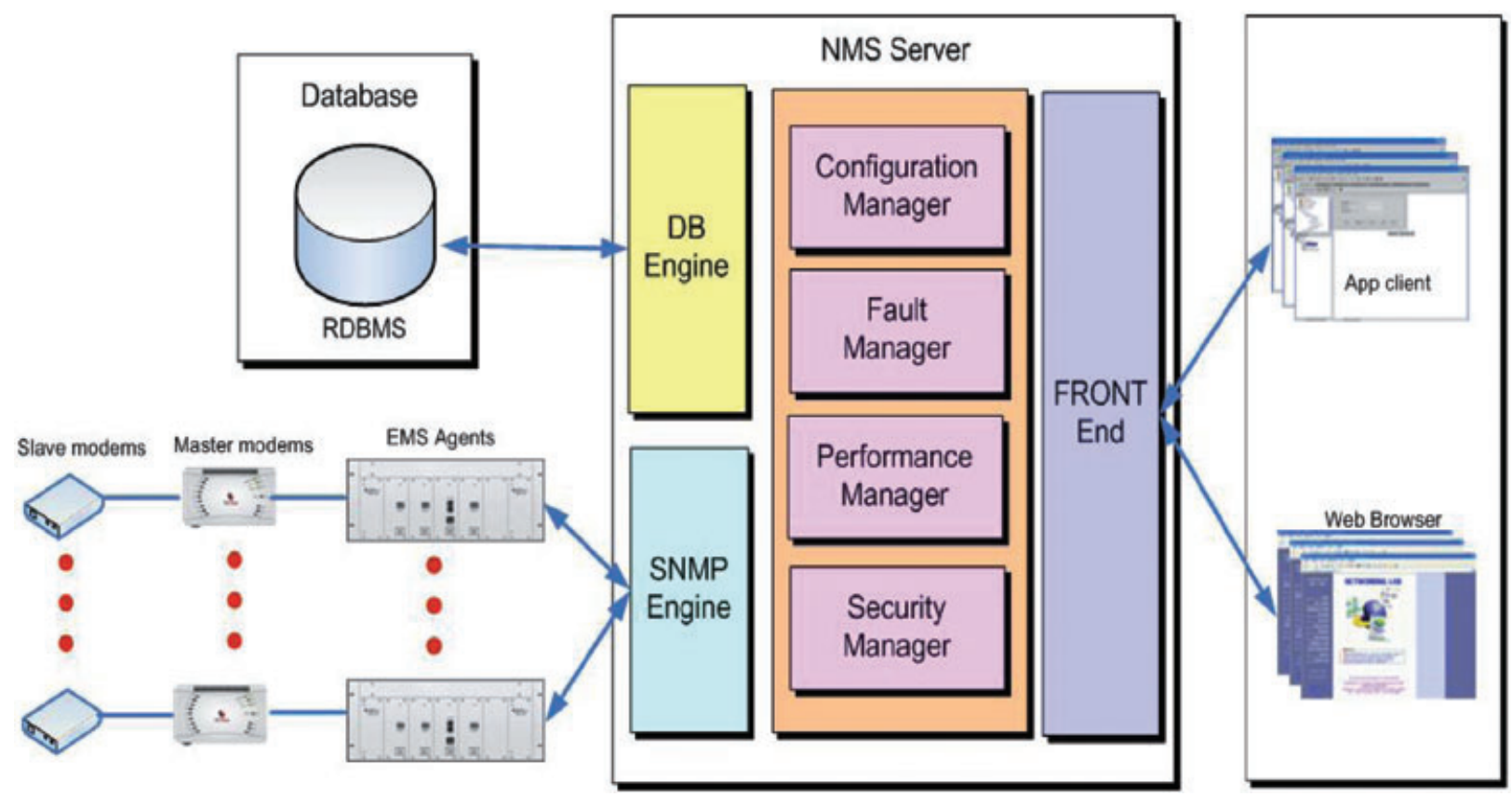

Figure 8. Architecture of PLC NMS 
- Front-end manager: the module to manage communication between the client and the server.

- $D B$ engine: the module to access the management information database.

- SNMP engine: the module to communicate with EMS agents.

- EMS agent: the proxy agent which performs management operations on behalf of the manager by communicating with the PLC-managed devices.

\subsection{Component design of PLC NMS}

In this section we present the component design of PLC NMS, which provides configuration management, performance management, fault management and security management.

\section{Configuration manager}

A PLC network administrator can monitor the state and control elements using configuration management functions. Hence, the network administrator can view all of the network states such as the state of links between the master and the slave modems. The configuration manager consists of three modules: the discovery module, which discovers managed network elements; topology module, which represents associations among network elements; and map module, which graphically shows managed network elements.

The discovery module gathers all the information of modems in the PLC networks. Here we deal just with medium- and low-voltage modems. Basically this module discovers the managed modems which are connected to management systems, and it can find all network elements or a part of them in the other networks by changing the environment setting file. For example, if the IP address of the PLC network management system is 172.28.110.xxx and the subnet mask is 255.255.255.0, the system discovers the modems in the 170.18.110.0. In terms of the change of the discovery environment setting files (i.e., seed, file), all managed nodes can be discovered.

The topology module shows the associations among the managed network elements. They are located in several sub-networks. We have generalized managed network elements to be managed through object modeling. Managed objects in PLC networks in our system have an IP address or group ID. The agent has an IP address and the modem and repeater have group ID + device ID. This means that modem and repeater have no IP address. In our system, we only manage PLC agent, PLC master modem, PLC slave modem and repeater. Group IDs for a modem range from 0 to 7 . Each group ID can include one master modem, three repeaters and 28 slave modems. Also we model the link between managed objects.

The map module plays a role in showing the topology map and managed objects as a graphical user interface (GUI). It represents the logical associations for networks, managing elements, and managed objects and their status. Therefore, a network operator can analyze the network status through it. Figure 9 shows the architecture of the map module. The map module consists of the map server, map DB, map session and clients. The map server maintains the map and provides maps to the client application by the map session. This initiates maps.config, map.filters and mapIcon.data files. In order to recognize changes in the objects, the map server delegates an observer to the topology module. This is managed in XML form and shows the detailed information to be presented to GUI, or map topology, current topology, the path of the tree icon file, and background image. Also, maps.filters and mapIcon.data files are generated and managed in XML form.

\section{Performance manager}

The performance manager for PLC networks gathers and analyzes the usage and error rates of the links. It also displays those matrices on the GUI. Using this information, an administrator can understand the state of the PLC networks in real time. Also, the administrator can set the threshold values to analyze the state of PLC networks on various assumptions. In order to collect, store, monitor and report, the performance manager has the managed objects and the data management policies defined in XML form. Managed objects that are related to the performance management define device name, type, owner, state, and data collection period. The polling object defines the data collected by the system and the device that 


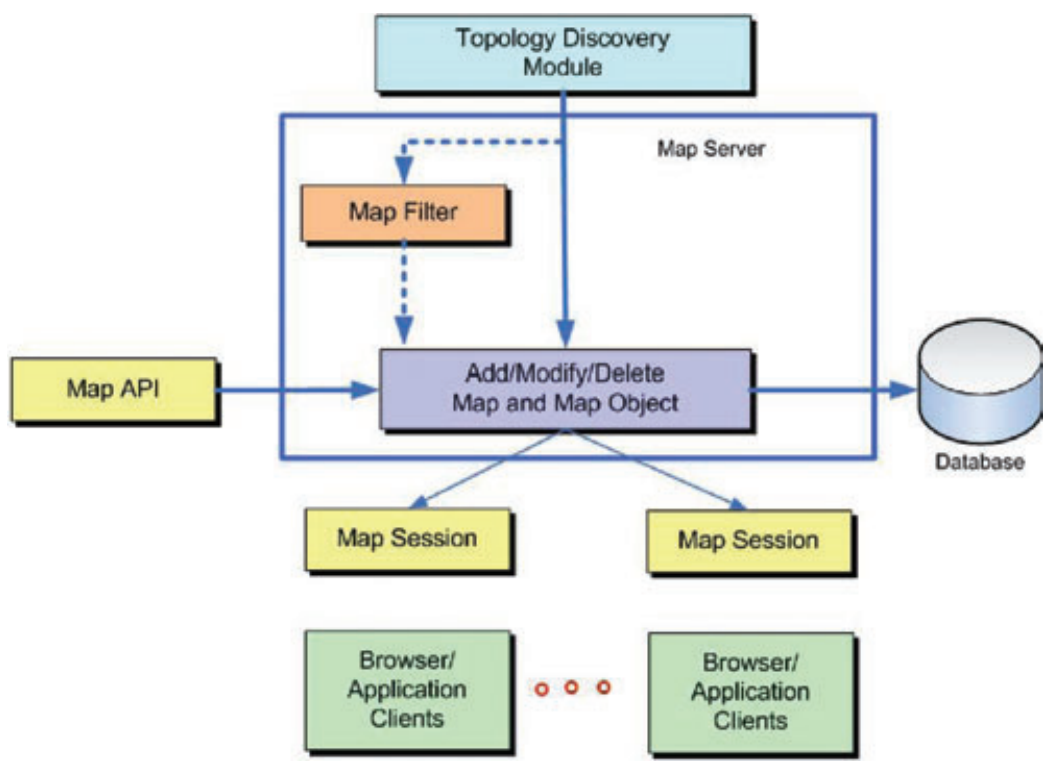

Figure 9. Architecture of configuration manager

collects the data. This object has two criteria in collecting data: data collection criteria and match criteria. The data collection criteria define the types of data that can be collected and also define agent name and collection period. The match criteria consist of one or more comparison statements, and they have properties, administrator, and set values. The threshold object describes the threshold values, its types, such as MIN, MAX, and EQUAL, and violation messages. If the data from PolledData object violate the threshold values, it notifies the administrator via the fault manager.

To filter the collected data from the managed object, the filtering policy defines addition to, modification to, and deletion from the polled data. The threshold policy defines the threshold value to prevent the performance degradation of the PLC networks. When there is a violation, the system generates the threshold event and notifies it to the fault manager. The report policy is used to describe the types of performance items to be generated on a daily, weekly, monthly and yearly basis. Also, it defines the minimum, maximum, and average values, per instance, to be presented in graphical form.

\section{Fault manager}

The fault states that are generated from PLC modems can be monitored in real time using the fault notification information or in non-real time using the stored data. For these functions, the fault manager uses SNMP traps and events generated from the performance manager by violating the defined threshold value. Our system defines the properties and operations of an event and an alert for the cooperation between them. Figure 10 illustrates the architecture of fault manager in our system. There are three events and predefined traps. The event and trap information are stored previously in the DB, which can be retrieved by the network administrators. The event information is designed so that email and SMS messages can be sent to the administrators.

The events are generated by the topology module and the performance module. The discovery event is generated for notification when a PLC modem is found in the networks, and the status polling event is generated by the result of periodic status data collection. In a normal state, the system generates a status up event (severity: clear), while, in abnormal state, it generates a status down event (severity: major). Moreover, the event by the performance module is the threshold event which is set by the performance module. If a PLC modem has a fault, an alert is generated. When the severity is neither in a normal state nor a 'clear' state, an alert is also generated. The action is for notifying the operation on the 


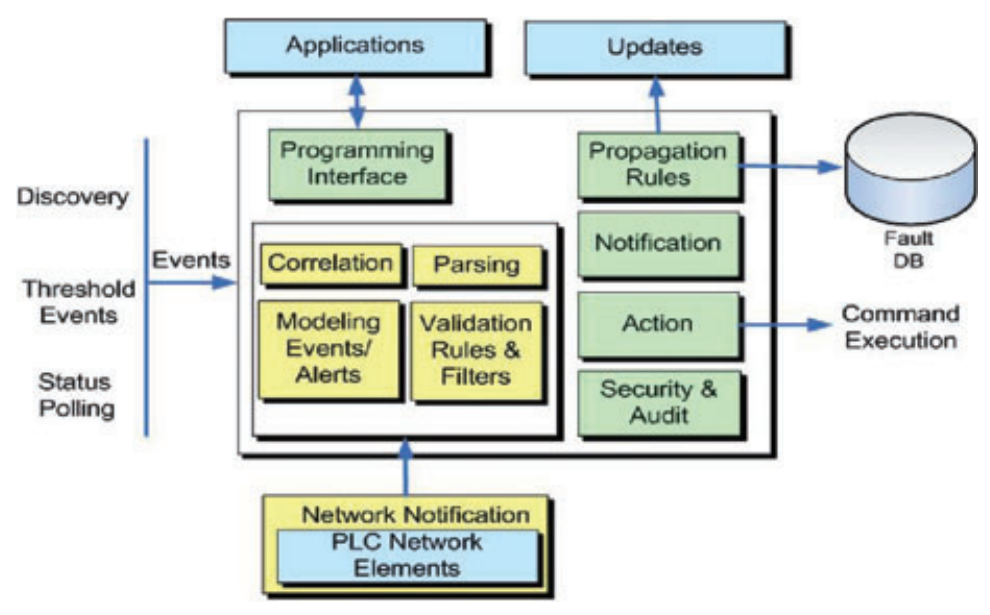

Figure 10. Architecture of fault manager

faulty state of the PLC modem to the administrator and displaying the state of severity of the faults using GUI.

\section{Security manager}

In order to ensure the secure operation of the network, such as data integrity, network availability, and authorized access, we designed a security manager. The security manager operates independently of configuration, performance, and fault management system and it is used to protect configuration, data collection, and event services. For example, any access trial to the NMS will be judged by the authentication policy part of the security manager under the user group mechanism. For each network administrator, the system can support security policy selection among the menu items. For securely managing the data, we use the RSA (Rivest Shamir Adleman) algorithm [15] and PKI both in access authentication and data encryption.

\section{IMPLEMENTATION OF PLC NETWORK MANAGEMENT SYSTEM}

We have implemented a PLC NMS with the design presented in the previous section using the Java platform [16] on an MS Windows platform. For the database, we used RDBMS which provides JDBC.

Figure 11 illustrates the topology map for PLC network elements. In the map, modems can be differentiated by group ID and station ID and identified by the icon view type as well. If we click the modem icon, it shows the performance parameters and QoS values for the master, the slave modems and repeaters, and averages the values on a daily and weekly basis.

If PLC nodes are discovered, the performance module collects the data according to the scheduling information. Performance parameters consist of the channel puncturing indicator, automatic gain control, bits per second, detected collisions, contention losses, discarded packets, transmitted packets, received packets, and so on. Basically, we collect the performance data every minute, and the GUI provides an example of the real-time graph. This system provides five types of graph, such as bar and line.

The fault manager mainly collects the alarm information for managing the health of PLC modems. The severity level and historical data of faults can be viewed for a specific node. The alarm information is related to the current fault information, which is different from the event view.

Security management supports the user and group-level mechanism. Also, it manages the accessibility levels for users. Users in a group can have a designated maximum access level. Audit trail function is used for recording the administrator's behaviors. 


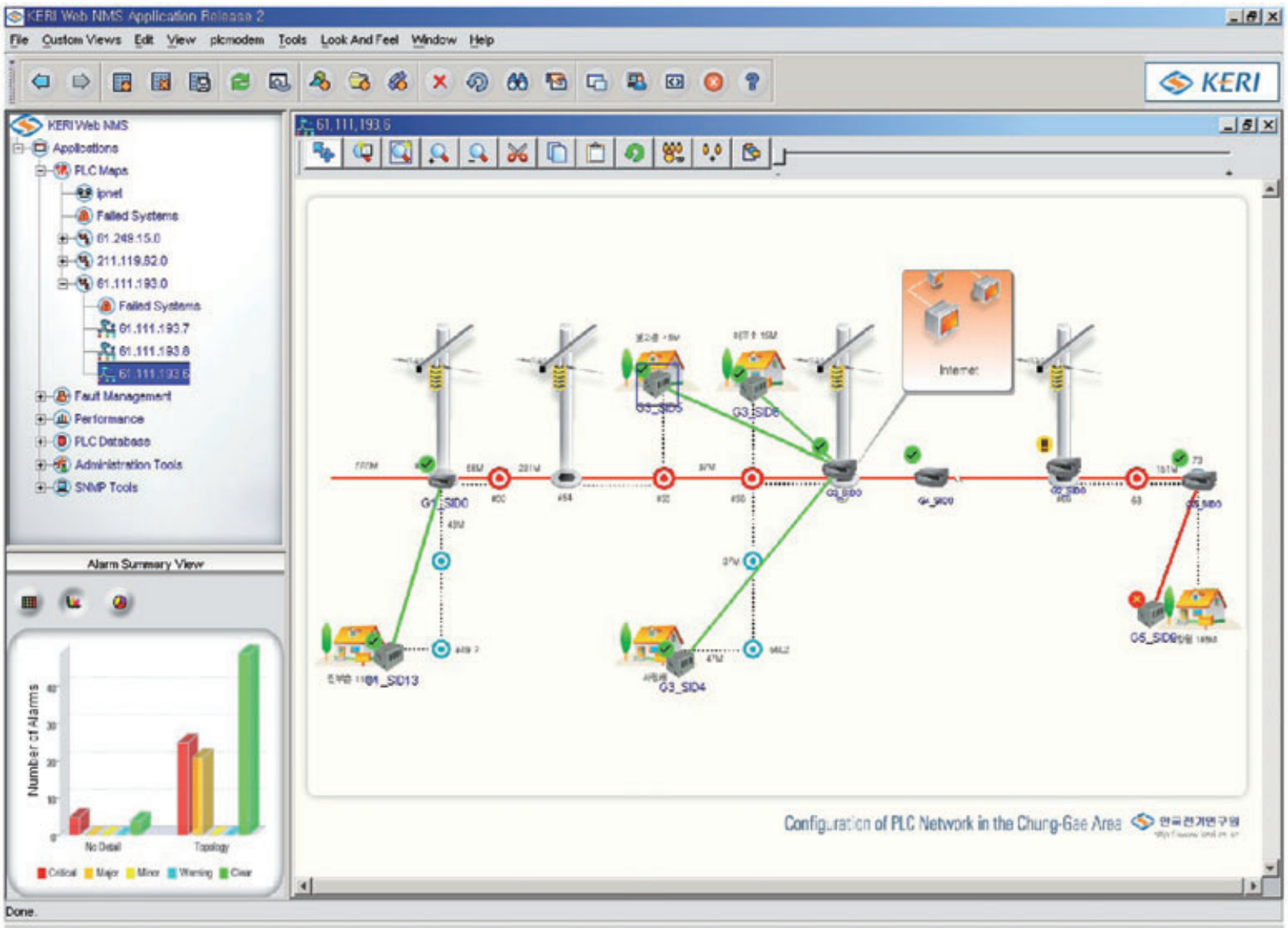

Figure 11. The topology map for PLC network elements

\section{EXPERIENCE}

In this section we present the result of the PLC trial network and service as well as its management using PLC NMS in the Chunggae area, located in the suburbs of Euiwang city near Seoul, Korea. As mentioned earlier, we have constructed the PLC subscriber network using MV and LV power lines. For Internet access and VoIP service to 20 households using the MV PLC network, a PLC subscriber network has been deployed.

Figure 12 shows the screen view of the configuration of the MV PLC subscriber network in the Chunggae area. The manager is made by the Web NMS platform, which is a management framework for building custom network management applications. As shown in Figure 12, the PLC subscriber network consists of the MV PLC network and LV PLC network.

All parameters for performance management described earlier can be stored, not only as current performance data but also historical performance data. Figure 13 shows the historical performance graph of the received bytes in a MV PLC modem; '-S(Station)' is the speed of downlink and '-SP(Station Parent)' is the speed of uplink. The original values of 'ReceivedCumulative- $x x^{\prime}$ MIB variables are cumulated. However, those in 'Received Bytes graph' are the results subtracted from the previous value. By default, the historical data of the last 24 hours are displayed. It can choose from the range of data such as today, last week, or even custom based. The usual service speed is approximately between 3 and $5 \mathrm{Mb} / \mathrm{s}$ at every service point. 


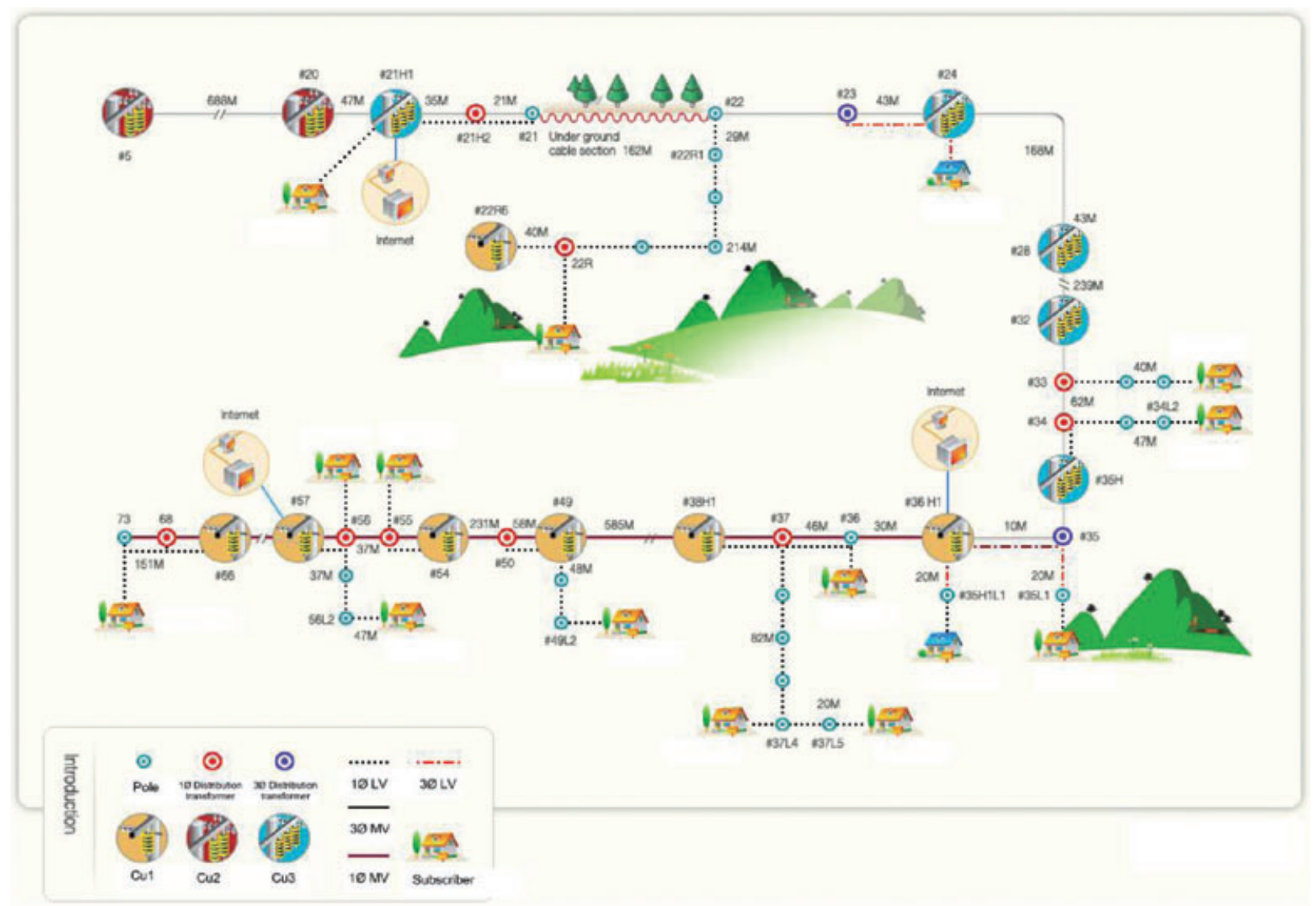

Figure 12. Deployment configuration of PLC network in the Chunggae area

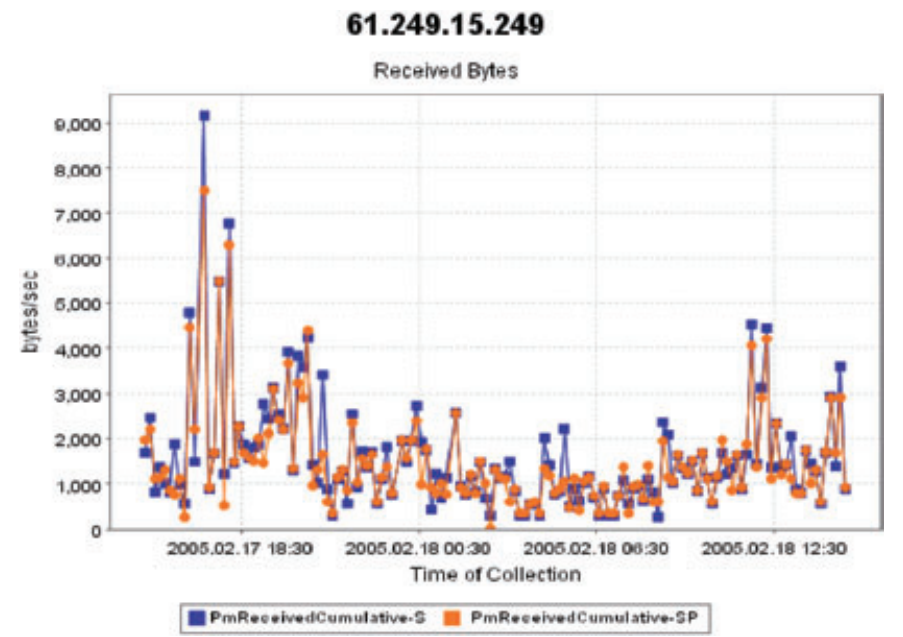

Figure 13. Performance graph of received bytes in MV PLC modem

The NMS can effectively manage a variety of data, but it can affect the performance of the whole PLC access network in a negative manner when we gather all the management parameters. Research into hierarchical parameter management is needed for the wide deployment of the PLC access network. Security is also essential and we need to develop a secure environment, considering the characteristics of PLC too. 


\section{CONCLUDING REMARKS}

Recently, PLC has received a lot of attention from both industry and academia. It is the area where more effort is needed to develop technologies for faster, interference-free, reliable and secure communication as well as for in-home networks using power lines. PLC is viewed as a cost-effective method for realizing ubiquitous networking and computing.

In this paper, we have briefly introduced the Korean PLC network technology and trial PLC network. We have also presented the design and implementation of PLC NMS for monitoring the trial network. Our experience so far indicates that Internet access and VoIP service is viable and as effective as the existing broadband Internet access technologies. One of the key challenges is to develop and deploy costcompetitive products.

We believe that PLC networking is viable not only for the access network but also for the in-home network. Perhaps there is more potential for PLC to succeed in the home networking area due to the fact that electrical wiring is available in most homes, and various home application devices (such as PCs, audio, video, camera, home appliances, etc.) can be easily networked via the PLC network. There is no doubt that we will see many more interesting applications using PLC networking technology in the next few years. The Korea Electricity Power Company (KEPCO) is planning to replace analog power meters with digital power meters for 20 million households in Korea by the year 2015. The technology that has been examined to be most promising for this replacement is PLC.

We are currently developing a home gateway which has several network access interfaces, including PLC, Wi-Fi, Ethernet, and Bluetooth. This gateway will enable various home networking services to interact and to remotely monitor and control home appliances and other devices. We plan to monitor and control these home appliances and devices through PLC networks and its management system. We also plan to develop a management system for a remote electricity metering trial service, which is being planned for 15000 households in Korea in 2007.

\section{ACKNOWLEDGEMENTS}

This research was supported by the Ministry of Information and Communication (MIC), Korea, under the Information Technology Research Center (ITRC) support program supervised by the Institute of Information Technology Assessment (IITA) (IITA-2005-C1090-0501-0018) and by the Electrical and Computer Engineering Division at POSTECH under the BK21 program of the Ministry of Education, Korea.

\section{REFERENCES}

1. Pavlidou N, Han Vinck AJ, Yazdani J. Power line communications: state of the art and future trends. IEEE Communications Magazine 2003; April: 34-40.

2. Majumder A, Caffery J. Power line communications. IEEE Potentials 2004; 23(4): 4-8.

3. Jee G, Rao RD, Cern Y. Demonstration of the technical viability of PLC systems on medium- and low-voltage lines in the United States. IEEE Communications Magazine 2003; 41(5): 108-112.

4. Liu W, Widmer H, Raffin P. Broadband PLC access systems and field deployment in European power line networks. IEEE Communications Magazine 2003; 41(5): 114-118.

5. IEEE. Standard for broadband over power line hardware. IEEE P1675. http://grouper.ieee.org/groups/bop/ [3 October 2006].

6. IEEE. Draft standard for broadband over power line networks: medium access control and physical layer specifications. IEEE P1901. http://grouper.ieee.org/groups/1901.

7. IEEE. Standard for powerline communication equipment: electromagnetic compatibility (EMC) requirements: testing and measurement methods. IEEE P1775. http://grouper.ieee.org/groups/bpl/ [3 October 2006].

8. OPERA (Open PLC European Research Alliance). http://www.ist-opera.org/ [3 October 2006].

9. UPA (Universal Powerline Association). http://www.upaplc.org [3 October 2006]. 
10. Xeline. http://www.xeline.com/ [3 October 2006].

11. DS2. http://www.ds2.es/ [3 October 2006].

12. Intellon. http://www.intellon.com/ [3 October 2006].

13. Lee J-J, Park Y-J, Kwon S-W, Oh H-M, Park H-s, Kim K-H, Lee D-Y, Jeong Y-H. High data rate Internet service over medium voltage power lines. In Proceedings of ISPLC 2005, April 2005; 405-408.

14. Stallings W. SNMP, SNMPv2, SNMPv3, and RMON 1 and 2 (3rd edn). Addison-Wesley: Reading, MA, 1999.

15. RSA. PKCS \#1 v2.1: RSA Encryption Standard, June 2002.

16. AdvenNet. http://www.adventnet.com/ [3 October 2006].

\section{AUTHORS' BIOGRAPHIES}

Jae-Jo Lee (jjlee@keri.re.kr) is a Senior Researcher in the Power Telecommunication Network Research Group, Electro-Fusion Research Division, Korea Electrotechnology Research Institute (KERI), Uiwang, Korea.

Choong Seon Hong (cshong@khu.ac.kr) is a Professor in the Dept. of Computer Engineering, Kyung Hee University, Seoul, Korea.

Joon-Myung Kang (eliot@postech.ac.kr) is a graduate student in the Dept. of Computer Science and Engineering, POSTECH, Pohang, Korea.

James Won-Ki Hong (jwkhong@postech.ac.kr) is a Professor in the Dept. of Computer Science and Engineering, POSTECH, Pohang, Korea. 